\title{
Single-molecule-based super-resolution images in the presence of multiple fluorophores
}

\author{
Paul D. Simonson, Eli Rothenberg ${ }^{1}$, and Paul R. Selvin ${ }^{2}$ \\ Physics Department and Center for Physics of the Living Cell, University of Illinois at Urbana- \\ Champaign, 1110 West Green Street, University of Illinois at Urbana-Champaign, Urbana, IL \\ 61801
}

\begin{abstract}
Several super-resolution techniques exist, yet most require multiple lasers, use either large or weakly emitting fluorophores, or involve chemical manipulation. Here we show a simple technique that exceeds the standard diffraction limit by $5-15 x$ on fixed samples, yet allows the user to localize individual fluorophores from among groups of crowded fluorophores. It relies only on bright, organic fluorophores and a sensitive camera, both of which are commercially available. Super-resolution is achieved by subtracting sequential images to find the fluorophores that photobleach (temporarily or permanently), photo-activate, or bind to the structure of interest in transitioning from one frame to the next. These fluorophores can then be localized via Gaussian fitting with selective frame averaging to achieve accuracies much better than the diffraction limit. The signal-to-noise ratio decreases with the square root of the number of nearby fluorophores, producing average single-molecule localization errors that are typically $<30 \mathrm{~nm}$. Surprisingly, one can often extract signal when there are approximately 20 fluorophores surrounding the fluorophore of interest. Examples shown include microtubules (in vitro and in fixed cells) and chromosomal DNA.
\end{abstract}

\section{Keywords}

super-resolution; microtubules; photobleaching; blinking; transient binding; gSHRImP; PAINT; DNA

\footnotetext{
Super-resolution fluorescence techniques, i.e., those that achieve resolution below the diffraction limit ( $\sim 250 \mathrm{~nm}$ ) have the potential to revolutionize microscopy, enabling one to see fine details in cell imaging. Two general classes of techniques exist. First are those that are fundamentally single-molecule localization techniques, such as (fluorescence) photoactivation localization microscopy ([f]PALM) ${ }^{1,2}$ and stochastic optical reconstruction microscopy (STORM) ${ }^{3}$. These rely on photactivatable fluorophores where a laser at one wavelength renders a fluorophore "activated" and a second laser is then used to excite fluorescence. The photoactivatable fluorophores can be expressed at high density, but less than one molecule per diffraction-limited area is usually active. The fluorophore's center can be localized to $<50 \mathrm{~nm}$ using fitting methods. Many fluorophores are localized to produce a

${ }^{2}$ To whom correspondence may be addressed: Telephone: (217) 244-3371, Fax: (217) 244-7559, selvin@illinois.edu. ${ }^{1}$ Present address: Department of Biochemistry, New York University School of Medicine, 550 First Avenue, MSB 363, New York, NY 10016

Supporting information available: additional, detailed descriptions of materials; experimental methods; figures; and information on algorithms and theoretical limits. This material is available free of charge via the internet at http://pubs.acs.org.

SUPPORTING CITATIONS

References 22232425262728 appear in the Supporting Material.
} 
composite super-resolution image. Another single-molecule technique, points accumulation for imaging in nanoscale topography (PAINT), relies on the stochastic binding of diffusing fluorophores that, once bound, can be localized ${ }^{4,5}$. Second are techniques like stimulated emission depletion (STED) ${ }^{6}$ and structured illumination microscopy (SIM) ${ }^{7}$ which do not rely on detecting single molecules but, rather, excite the sample using special illumination techniques. These techniques typically achieve $<100 \mathrm{~nm}$ resolution (for review, see ${ }^{8}$ ). An in-between technique, super-resolution optical fluctuation imaging (SOFI) relies on the blinking of single fluorophores, but the analysis involves calculating the statistical moments of the pixels rather than localizing single fluorophores ${ }^{9}$.

Despite their importance, these methods often include requirements that make it somewhat complex for the typical laboratory to apply. For example, STORM and PALM require photoactivatable fluorophores and laser cycling equipment. SIM requires very specialized optics, while STED requires sensitive alignment of optics and high laser excitation powers. More recent techniques have overcome some of these problems. For example, ground-state depletion followed by individual molecule return (GSDIM), uses relatively high excitation intensities to force the majority of molecules into metastable dark states. This achieve a low densities of actively fluorescing molecules, but is also compatible with many conventional fluorophores ${ }^{10}$. dSTORM ${ }^{11}$ and other similar blinking microscopies ${ }^{12}$ use additional chemicals to cause fluorophores to blink with long-lived dark states and remove the necessity of laser cycling. SOFI in fact does an excellent job of producing super-resolution images from ensembles of simultaneously active fluorophores that blink significantly for rather long periods of time (like quantum dots). In general, however, these techniques require special optics, special chemicals, or have limitations in terms of what can be labeled.

We desired to create super-resolution images using standard fluorophores and normal imaging techniques. To do this, it was necessary to develop an analysis approach that would allow us to localize single fluorophores, even within a bright, complex fluorescent background ${ }^{1}$. The approach is similar to single molecule high resolution imaging with photobleaching (SHRImP), which handles two fluorophores with overlapping, diffractionlimited spot images ${ }^{13}$, or the equivalent technique, nanometer-localized multiple singlemolecule fluorescence microscopy (NALMS), which handles up to $\sim 5$ fluorophores ${ }^{14}$. Our approach, which we call generalized SHRImP (gSHRImP), generalizes the approach to handle thousands of fluorophores with up to about 20 fluorophores per diffraction-limit area. We find a 5-20x increase in resolution by relying on natural photobleaching and photoactivating. Surprisingly, one can avoid getting "lost in the noise" despite the presence of surrounding fluorophores.

\section{gSHRImP analysis}

(An overview of gSHRImP analysis as it is applied to a simple two-fluorophore system is shown in Fig. 1.) A fluorescent movie is taken, during which time the fluorophores naturally undergo photobleaching (permanent or temporary) and possibly photoactivation. A frame with $(n-1)$ fluorescing molecules is subtracted from a preceding image that contains $n$ fluorescing molecules to produce the "subtracted" image. A spot will then be present in the resulting image that corresponds to the photobleaching event. The spot is automatically detected and localized to pixel accuracy by finding the local maxima of the intensity. (See Supplemental Text). The spot is then further localized to sub-pixel accuracy using a twodimensional Gaussian fit. This is repeated with the $(n-2)$ image subtracted from the $(n-1)$ image, etc, until all the molecules are photobleached.

Overcoming the photon noise is of course critical. The situation here is much like an absorption measurement that is inherently noisier than a fluorescence measurement because 
one is measuring "down" from a high-level, instead of "up" from a near-dark background. Averaging frames both before and after the photobleaching event reduces the localization error. In particular, the noise due to photon statistics is reduced by a factor of $\sqrt{F}$ where $F$ is the number of frames averaged. The specific number of frames to be averaged is found by searching for nearby photobleaching or photobleaching events in time and space, where the averaging is not necessarily the same for each event. The number of consecutive frames averaged before a photobleaching/activation event is the number of intervening frames between the event of interest and the preceding event that is located within a radius of 5-6 pixels; the frames averaged after an event are similarly chosen (see Fig. 1 for an example). For the data presented here, the maximum allowable number of frames to average was set to 20. Choosing a value larger than this is feasible, but then microscopy stage drift may need to be considered.

Spots that are not fit well by Gaussian functions (i.e., localization error is too large, spots are too narrow, spots are too wide, ellipticity $>1.5$, etc.) are rejected. (See the supplemental information for example parameters used for spot rejection). The accepted localizations are then used to plot a composite super-resolution image. The spot localizations are plotted as two-dimensional Gaussian functions with widths corresponding to the localization errors of the individual fits. (For more details of the analysis, see the Supplementary Text.)

An estimate of the localization error, $\sigma_{i}$, along a single axis in the $x-y$ imaging plane is given by Thompson et al. ${ }^{15}$

$$
\sigma_{i}=\sqrt{\frac{s^{2}+a^{2} / 12}{N}+\frac{8 \pi s^{4} b^{2}}{a^{2} N^{2}}}
$$

where $s$ equals approximately half the width of a Gaussian fit to the PSF; $a=100 \mathrm{~nm}$ (the pixel size); $N=$ total number of photons collected from a fluorophore; and $b=$ background noise. Under reasonable assumptions (see Supplementary Text),

$$
b=\frac{a \sqrt{m N}}{2 s}
$$

where $m=$ number of fluorophores in a diffraction-limited area and $N$ is the average number of total photons per fluorophore. If $F=$ number of frames that can be averaged before and after the photobleaching event, then the Thompson equation can be modified to (see Supplementary Text)

$$
\sigma_{i}=\sqrt{\frac{s^{2}+a^{2} / 12}{N}+\frac{4 \pi s^{2}(m-1)}{F N}} .
$$

Thus, if $s_{i}=143 \mathrm{~nm}, a=106.67 \mathrm{~nm}, N=1800$ photons, $m=10$, and $F=1$, then $\sigma_{i}=36 \mathrm{~nm}$. Increasing to $F=2$ gives $\sigma_{i}=25 \mathrm{~nm}$ (these values are typical of fluorophores localized in Fig. 2). For $\mathrm{m}=25$ and $\mathrm{F}=2, \sigma_{i}=41 \mathrm{~nm}$. Of course, the resolution will depend on when each molecule photobleaches and the emission intensity. In fact, Thompson et al, assumed a flat background, whereas we do not, and instead of using the equation above, we use the localization error calculated using a two-dimensional Gaussian fit (for more on the Gaussian fitting and error value estimate, see Supplementary Text). Of course, the photobleaching and blinking rates also determine the maximum density of fluorophores for a given frame rate allowed since photobleaching and/or photoactivation events should not occur simultaneously 
in time and space. Expressions for maximum fluorophore densities based on photobleaching and blinking rates are derived in the supplementary text.

Another approach for producing gSHRImP-compatible data is using diffusion-based labeling, as in PAINT ${ }^{4}$. (In fact, gSHRImP works equally well with photoactivation, blinking, fluorophore binding, etc.- -all that matters is that single fluorophores appear to transition between dark and bright states or between bright and dark states.) A low density of bound and fluorescing molecules can be achieved by imaging a sparsely-labeled object where the object is in a solution containing fluorescently-labeled molecules. The fluorophores are designed to be able to bind to the object of interest, either transiently or permanently. The single frame acquisition time is chosen to be slow compared to the time required for a fluorophore to diffuse through the length of an image pixel. Thus, fluorophores in solution contribute a diffuse fluorescent background, while immobilized fluorophores contribute a strong, localized fluorescent signal. Total internal reflection fluorescence (TIRF) microscopy can be used to further reduce background fluorescence from diffusing fluorophores. The bound fluorophores eventually photobleach or unbind, which helps to maintain a low density of bound, active fluorophores. If the labels bind irreversibly, the target should be initially unlabeled to maximize fluorophore localizations and accuracy. Combining PAINT with gSHRImP allows imaging with higher fluorophore densities (because fluorophore PSFs can overlap; this can also quicken the speed at which a given number of fluorophores can be localized) and thus requires less optimization of fluorophore concentrations than for PAINT alone.

We applied gSHRImP to several fluorophore-labeling systems and dynamics (photobleaching, blinking, and binding/unbinding fluorophores) on a variety of systems-microtubules, axonemes, and DNA, outlined below.

\section{gSHRImP applied to photobleaching microtubules in vitro}

In vitro microtubules labeled with tetramethylrhodamine (TMR) were photobleached under TIRF microscopy. In a normal, diffraction-limited image, the microtubules' full width at half maximum (FWHM) is $300 \mathrm{~nm}$ (Fig. 2b, d), even though the true microtubule diameter is $24 \mathrm{~nm}$. The super-resolution image found using gSHRImP analysis produced a FWHM of $60 \mathrm{~nm}$ (Fig. 2c-d, see also Fig. S1). Given that the diameter of the microtubule is $24 \mathrm{~nm}$ and the average localization error of single spots (i.e., the average error associated with localizing the center of an individual fluorophore) is $\sim 20 \mathrm{~nm}, 60 \mathrm{~nm}$ corresponds well within the expected value. Fluorophore blinking played an important role in creating the superresolution image (see Table S1).

\section{gSHRImP applied to photobleaching microtubules in fixed cells}

We also imaged microtubules in fixed COS-7 cells, labeled with primary antibodies and secondary antibodies conjugated to TMR, Alexa Fluor 647, or CF633. The regular TIRF images gave microtubule widths near $500 \mathrm{~nm}$. (Note that antibody-labeling increases the width, and microtubules were not fit as well to straight lines in this case as in the in vitro case). With all three dyes, we found significant improvement in resolving microtubules using gSHRImP. We found our best results using CF633 (see Fig. 3), followed by TMR. This is likely due to the excellent photostability and brightness of the CF633 dye. Again, we found a significant portion of the fluorophore localization was due to blinking (see Table S2). The average localization error of plotted spots was $17 \mathrm{~nm}$, and the FWHM of microtubule images was $100 \mathrm{~nm}$. We also imaged microtubules in cells by transfecting with eGFP-tubulin. Unfortunately, eGFP was not bright enough, nor stable enough, to use in gSHRImP. 


\section{gSHRImP applied to photobleaching axonemes}

Isolated axonemes are expected to have a diameter between 160 and $200 \mathrm{~nm}$, depending on the presence of $\mathrm{Mg}^{++}$in the surrounding buffer ${ }^{16}$. Because of the large diameter and the short penetration depth of excitation light into samples using TIRF microscopy, it was necessary to image axonemes using non-TIRF illumination. We labeled the axonemes by directly conjugating them to TMR. For regular microscopy, the FWHM was $400 \mathrm{~nm}$ (Fig. S2). The FWHM using gSHRImP was $180 \mathrm{~nm}$ (Fig. S2). This is 2.2 times smaller than that of regular microscopy. Also, the width in the gSHRImP image is close to the expected value of the axoneme diameter.

\section{gSHRImP with fluorescent paclitaxel}

Microtubules were imaged in a $7 \mathrm{nM}$ solution of fluorescent Oregon Green 488 paclitaxel (Invitrogen, Cat. no. P22310). Paclitaxel, also known by the trade name "Taxol," is an anticancer drug that binds to microtubules to inhibit cell division. At low concentrations in TIRF microscopy, background fluorescence is relatively low. The average localization accuracy of a single dye was $31 \mathrm{~nm}$, as determined by gSHRImP (see Fig. 4a-b). Unfortunately, it was found that as the experiment continued for only a few minutes, the dyes rather suddenly became dim. Bright fluorescence was restored after flowing in fresh imaging solution. Further imaging experiments suggested that the problem involved the PCA-PCD deoxygenation system, which was used for prolonging the photobleaching lifetime of the dyes.

\section{gSHRImP with mutant-streptavidin}

We also investigated streptavidin as a general-use protein for transient labeling. Transient labeling is desirable because photobleached fluorophores can in principle be replaced indefinitely by new fluorophores, allowing imaging ad infinitum. To reduce biotin-binding affinity (native binding is nearly irreversible) we used streptavidin that was mutated at a single amino acid (S45A, compliments of P.S. Stayton ${ }^{17}$ ). This mutation increases the dissociation constant by 1700 fold, leading to a bound state lifetime of $14 \mathrm{~s}$ at $37^{\circ} \mathrm{C}^{17}$. By using streptavidin S45A, it is then possible to transiently bind biotinylated fluorophores to any biotinylated target.

As an example, biotinylated microtubules were attached to kinesin-coated glass. $40 \mathrm{~nm}$ dark-red fluorescent beads were added to the sample to serve as fiduciary markers. The microtubules were imaged in $52 \mathrm{nM}$ streptavidin S45A and $13 \mathrm{nM}$ double-stranded DNA oligomers conjugated to biotin at one end and Atto647N at the other (see Supplemental Text). Fig. 4c-e shows the result after gSHRImP analysis. The average fluorophore localization error was $9 \mathrm{~nm}$ (note that in this case, background tended to be quite low). Despite this success, the on-rate of the fluorophores to the microtubules gradually decreased over tens of minutes and could not be attributed to depletion of active fluorophores in solution. Transiently-binding DNA oligomers could possibly be used as an alternative approach to achieve transient labeling ${ }^{18}$.

\section{gSHRImP applied to DNA}

Finally, we applied the PAINT-gSHRImP method to image DNA. Lambda DNA was flowed over a poly-L-lysine coated coverslip and imaged. DNA appeared as lines on the surface. We used a weak ( 10 nM) solution of SYTO 16 in $3 \mathrm{mg} / \mathrm{mL}$ BSA and phosphate buffered saline (PBS) while imaging. Adding BSA appeared to slow the diffusion rate of the dyes so that binding events were more distributed in time. We started imaging the DNA shortly after the dye was added so that many of the binding sites were not yet occupied at 
the beginning of data acquisition. Resulting average fluorophore localization error was 36 nm (Fig. S3).

We also applied the technique to DNA in cells. HEK 293 and HeLa cells were seeded on glass-bottom petri dishes, fixed with $20^{\circ} \mathrm{C}$ methanol for 10 minutes, and imaged in dilute solutions of DNA dye (Fig. 5). In this case, we used POPO-3, SYTO 16, and YOYO-1 dyes at nanomolar concentrations and $3 \mathrm{mg} / \mathrm{mL}$ BSA in PBS (see Figs. 5 and S4). The average localization error of single POPO-3 fluorophores in Fig. $5 \mathrm{c}$ was $33 \mathrm{~nm}$. This represents a new method for super-resolution imaging of chromosomal DNA in a cell.

\section{Discussion}

Using bright fluorophores and a sensitive camera, biologists are no longer limited by the diffraction limit of light. The gSHRImP analysis approach enables super-resolution imaging with an ensemble of molecules within a diffraction-limited area. This includes denselylabeled structures undergoing simple photobleaching; blinking fluorophores with overlapping spot images; fast, transient labeling with diffusing fluorophores; etc.; as demonstrated in Figs. 2-5. Because spots can overlap, the technique requires less concentrations optimization to produce super-resolution images. The technique is also compatible with any fluorophore that is sufficiently stable and bright. We found commercial DNA dyes of particular interest, which, as we have shown, allowed us to perform chromosomal DNA super-resolution imaging in cells using transient-labeling gSHRImP.

In the case that fluorophores bind transiently to the target, transient-labeling with gSHRImP provides particular advantages over other super-resolution techniques. Photobleached fluorophores can dissociate from the binding site and be replaced by non-photobleached fluorophores. In other techniques, permanently spent fluorophores cannot be replaced. Another advantage is that potentially every solvent-exposed binding site can be localized. This is often not possible using other techniques because of (1) the finite size of labeling antibodies or fluorescent proteins that block nearby binding sites, (2) pre-photobleaching of labeling fluorophores that bind but do not release, (3) separation distance requirements based on fluorophore-fluorophore interactions like quenching, etc. The diffusion-based labeling scheme is especially useful if the fluorophore does not fluoresce until it binds to the target, such as in the case of the DNA-binding fluorophores. Then, background fluorescence that would normally be contributed by diffusing fluorophores is eliminated. Combining transient labeling with gSHRImP simplifies the experimental part of the approach and can make numbers of spots localized per unit time faster than using transient labeling alone.

SHRImP does have limitations. In cases where the fluorophore binds and does not release, the method is limited by a maximum density of fluorophores. This is particularly important in the case of simple-photobleaching imaging where the density of active fluorophores can be very high. First, the dynamic range of the camera limits the maximum density. All cameras have a maximum light intensity at which the pixels become saturated. By lowering gain settings of the camera, the amount of light that can be detected without saturating the camera is increased, but the detection sensitivity is decreased. When the camera gain is too low, the signal-to-noise ratio for single fluorophores is too low, rendering the localization of individual fluorophores with high accuracy impossible. Second, if two (or more) fluorophores simultaneously photobleach (that is, on the order of the frame acquisition time of the camera), and if these fluorophores are close enough in space such that their PSFs overlap, then the two photobleaching events cannot be used to construct the final super resolution image (see also Supplemental Text). The density of fluorophores is therefore limited by the photobleaching rate of the individual fluorophores (or, in the case of transientlabeling, the concentration and on/off rates of labels in solution). It should also be noted that 
the approach is different from techniques such as DAOSTORM ${ }^{19}$ and similar deflation techniques that are aimed at speeding up image processing by localizing crowded fluorophores in single frames. However, gSHRImP does allow nearby fluorophores to be distinguished and localized that are closer than the minimum separation distances associated with DAOSTORM. Finally, unlike PALM, STORM, etc., in which the background noise due to nearby fluorophores is basically zero, gSHRImP does not require the background to be zero, and, as a result, the localization error due to noise will be increased. For these reasons, in simple photobleaching imaging (see Figs. 2 and 3), the density of fluorophores localized will usually be lower than that required to meet the Nyquist criterion, resulting in discontinuous-looking images; however, the simplicity of the technique, and known geometries of some structures (e.g., microtubules), make meeting the Nyquist criterion of lesser importance.

Thus, notwithstanding some limitations, we have introduced a robust technique that makes super-resolution using many more experimental methods possible. The technique is robust in the sense that it can be applied to data taken using a variety of experimental methods including photo-activation, blinking, diffusive and transient labeling, simple photobleaching, etc., or even a combination of all of the techniques. It is also robust in that overlapping spot images and background fluorescence (e.g., cell autofluorescence) are dealt with such that previously unusable data are now usable. Additionally, we have presented methods for transient labeling, including methods for labeling microtubules, biotinylated structures, and chromosomal DNA in a cell. Given that only recently has DNA been imaged at superresolution (see ${ }^{20,21}$ ), this latter application may be particularly important. The labeling and analysis methods demonstrated are straightforward to perform. These should make it possible for a larger number of investigators to handle fluorescent backgrounds and apply super-resolution imaging using techniques, commercial dyes, and equipment that are already found in many labs.

\section{Supplementary Material}

Refer to Web version on PubMed Central for supplementary material.

\section{Acknowledgments}

We thank Vladimir Gelfand for useful suggestions on how to label microtubules using antibody staining. This work was supported by NIH grant GM086214 and by NSF grants DBI-02-15869, EAGER 0968976, and 082265.

\section{REFERENCES}

1. Betzig E, Patterson GH, Sougrat R, Lindwasser OW, Olenych S, Bonifacino JS, Davidson MW, Lippincott-Schwartz J, Hess HF. Science. 2006; 313(5793):1642-1645. [PubMed: 16902090]

2. Hess ST, Girirajan TPK, Mason MD. Biophys. J. 2006; 91(11):4258-4272. [PubMed: 16980368]

3. Bates M, Huang B, Dempsey GT, Zhuang X. Science. 2007

4. Sharonov A, Hochstrasser RM. Proc. Natl. Acad. Sci. U.S.A. 2006; 103(50):18911-18916. [PubMed: 17142314]

5. Giannone G, Hosy E, Levet F, Constals A, Schulze K, Sobolevsky AI, Rosconi MP, Gouaux E, Tampe R, Choquet D, Cognet L. Biophys. J. 2010; 99(4):1303-1310. [PubMed: 20713016]

6. Hell SW. Science. 2007; 316(5828):1153-1158. [PubMed: 17525330]

7. Schermelleh L, Carlton PM, Haase S, Shao L, Winoto L, Kner P, Burke B, Cardoso MC, Agard DA, Gustafsson MG, Leonhardt H, Sedat JW. Science. 2008; 320(5881):1332-1336. [PubMed: 18535242]

8. Huang B, Bates M, Zhuang X. Annu. Rev. Biochem. 2009; 78:993-1016. [PubMed: 19489737] 
9. Dertinger T, Colyer R, Iyer G, Weiss S, Enderlein J. Proc. Natl. Acad. Sci. U.S.A. 2009; 106(52): 22287-22292. [PubMed: 20018714]

10. Folling J, Bossi M, Bock H, Medda R, Wurm CA, Hein B, Jakobs S, Eggeling C, Hell SW. Nat. Methods. 2008; 5(11):943-945. [PubMed: 18794861]

11. Heilemann M, van de Linde S, Mukherjee A, Sauer M. Angew. Chem. Int. Ed. Engl. 2009; 48(37): 6903-6908. [PubMed: 19670280]

12. Vogelsang J, Steinhauer C, Forthmann C, Stein IH, Person-Skegro B, Cordes T, Tinnefeld P. ChemPhysChem. 2010; 11(12):2475-2490. [PubMed: 20632356]

13. Gordon MP, Ha T, Selvin PR. Proc. Natl. Acad. Sci. U.S.A. 2004; 101(17):6462-6465. [PubMed: 15096603]

14. Qu X, Wu D, Mets L, Scherer NF. Proc. Natl. Acad. Sci. U.S.A. 2004; 101(31):11298-11303. [PubMed: 15277661]

15. Thompson RE, Larson DR, Webb WW. Biophys. J. 2002; 82(5):2775-2783. [PubMed: 11964263]

16. Warner FD. J. Cell Biol. 1978; 77(3):R19-R26. [PubMed: 150425]

17. Hyre DE, Le Trong I, Freitag S, Stenkamp RE, Stayton PS. Protein Sci. 2000; 9(5):878-885. [PubMed: 10850797]

18. Steinhauer C, Jungmann R, Sobey TL, Simmel FC, Tinnefeld P. Angew. Chem. Int. Ed. Engl. 2009; 48(47):8870-8873. [PubMed: 19830751]

19. Holden SJ, Uphoff S, Kapanidis AN. Nat. Methods. 2011; 8(4):279-280. [PubMed: 21451515]

20. Flors C, Ravarani CN, Dryden DT. ChemPhysChem. 2009; 10(13):2201-2204. [PubMed: 19554598]

21. Flors C. Photochem. Photobiol. Sci. 2010; 9(5):643-648. [PubMed: 20442922]

22. Aitken CE, Marshall RA, Puglisi JD. Biophys. J. 2008; 94(5):1826-1835. [PubMed: 17921203]

23. Howarth M, Chinnapen DJ, Gerrow K, Dorrestein PC, Grandy MR, Kelleher NL, El-Husseini A, Ting AY. Nat. Methods. 2006; 3(4):267-273. [PubMed: 16554831]

24. Lemons, DS.; Langevin, P. An introduction to stochastic processes in physics : containing "On the theory of Brownian motion" by Paul Langevin, translated by Anthony Gythiel. Baltimore: Johns Hopkins University Press; 2002.

25. Taylor, JR. An Introduction to Error Analysis: the Study of Uncertainties in Physical Measurements. Vol. Vol. 327. Sausalito, California: University Science Books; 1997.

26. Bos, Avd. Parameter estimation for scientists and engineers. Hoboken, N.J.: Wiley-Interscience; 2007. p. xiv273 p.

27. Smith CS, Joseph N, Rieger B, Lidke KA. Nat. Methods. 2010; 7(5):373-375. [PubMed: 20364146]

28. Ober RJ, Ram S, Ward ES. Biophys. J. 2004; 86(2):1185-1200. [PubMed: 14747353] 


\section{The gSHRImP algorithm}

Simple example:

1. Start with a two-fluorophore image sequence with photobleaching (frames 4 and 6):
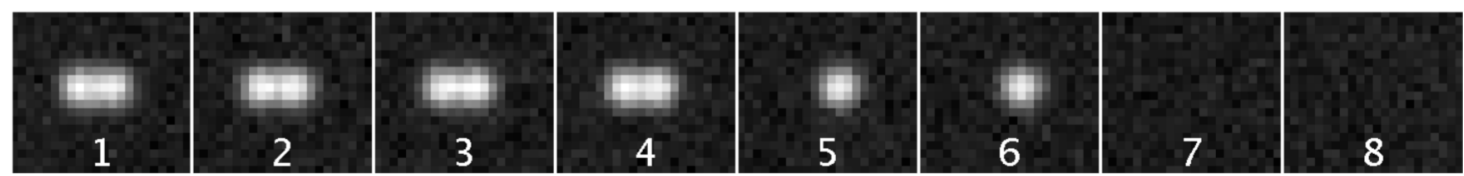

2. Create the backwards-subtracted image sequence by subtracting frame 8 from 7 to get new frame 7', 7 from 6 to get new 6', etc.:
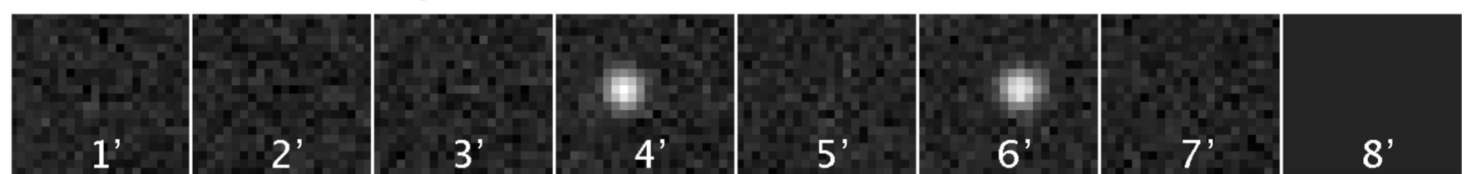

3. Locate spots in the backwards-subtracted image sequence. These show when and where photobleaching occurred (here, frames $4 \& 6$ ):
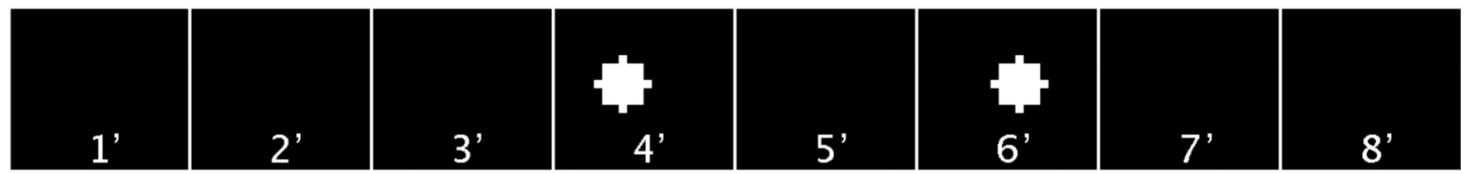

4. Localize the first photobleaching fluorophore by subtracting the average of frames 5-6 from the average of frames 1-4, then fitting to a weighted 2-dimensional Gaussian:

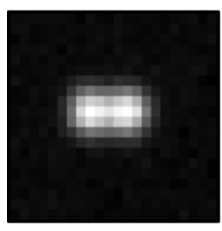

Average of $1-4$

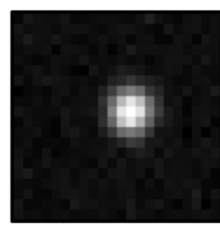

Average of 5-6

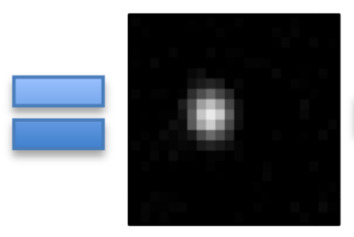

Image for Gaussian fitting

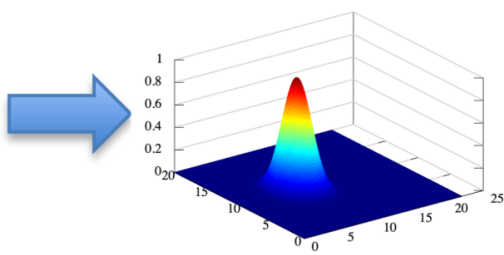

Gaussian Fit

5. The center of the Gaussian fit is the location (within fit error) of the fluorophore. Repeat for the second fluorophore but subtract the average of frames 7-8 from the average of 5-6.

6. Plot the locations of the fluorophores in the new, super-resolution image.
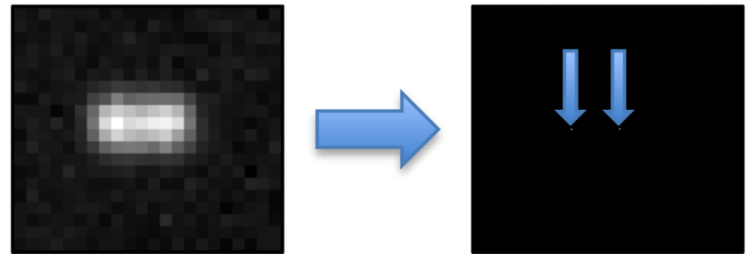

Figure 1.

Example of the gSHRImP algorithm applied to a simple, two-fluorophore movie. In a completely analogous way, fluorophores going from a dark state to a fluorescent state (as occurs with blinking fluorophores, photo-activation, or fluorophore binding events) are also localized. 

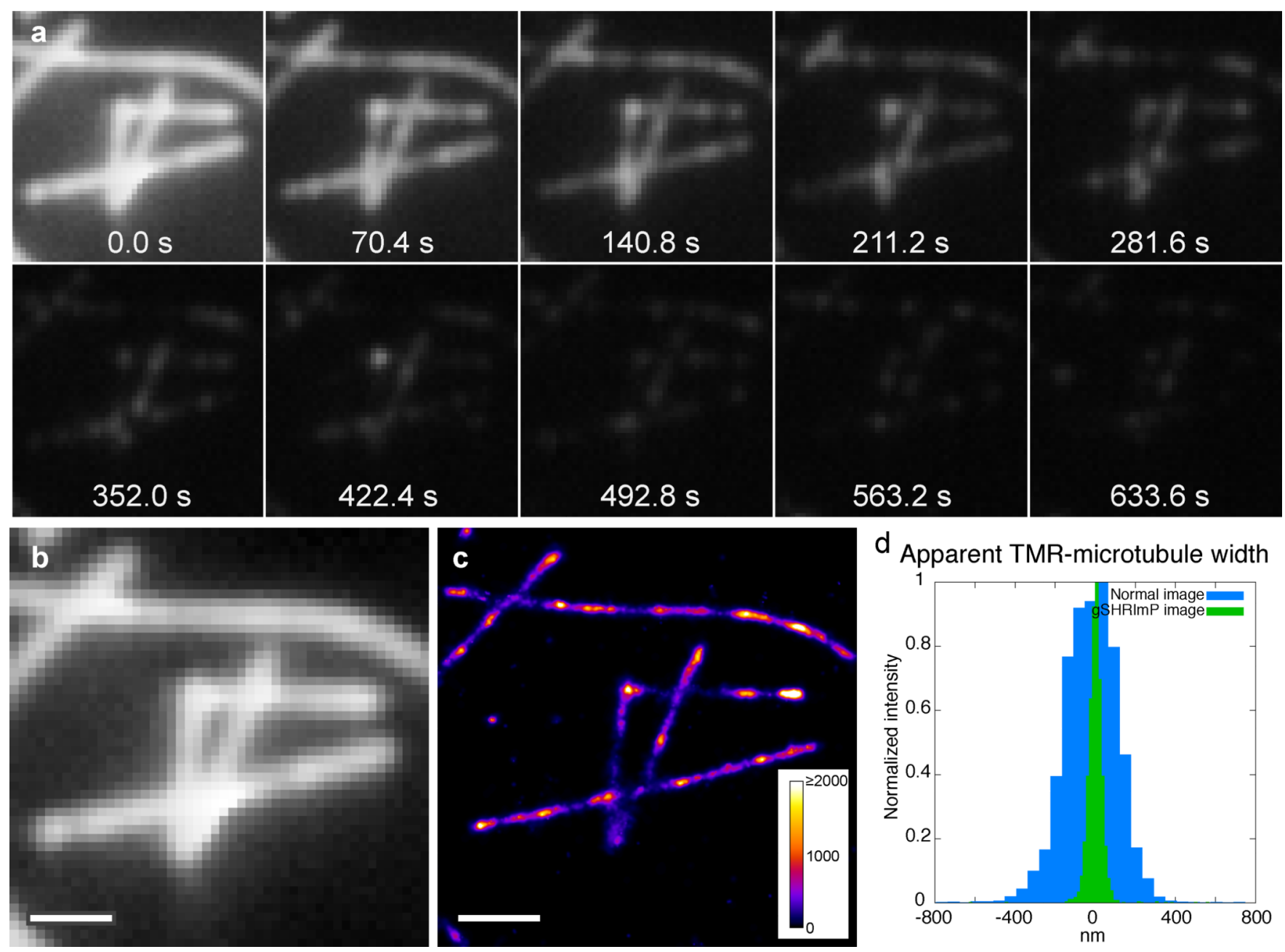

d Apparent TMR-microtubule width

Figure 2.

Application of gSHRImP to photobleaching TMR-labeled microtubules. (a) Selected frames from the total acquired movie (3178 total frames, $0.2 \mathrm{~s}$ per frame) demonstrating photobleaching and blinking. (b) Standard, diffraction-limited image. Scale bar $=1 \mu \mathrm{m}$. (c) Corresponding super-resolution image constructed using gSHRImP. Pixel length $=10 \mathrm{~nm}$. (d) Comparison of the microtubule FWHMs. In the normal image the microtubule FWHM is about $300 \mathrm{~nm}$, while in the gSHRImP image the microtubule FWHM is about $60 \mathrm{~nm}$. In part "c" the image was constructed using maximum likelihood fitting (see supplement on fitting; average estimated spot localization error $=27 \mathrm{~nm}$ ), while in "d" the data were analyzed using least-squares fitting (average estimated localization error $=20 \mathrm{~nm}$ ), although actual outcomes were very similar. See supplemental text for more details. 

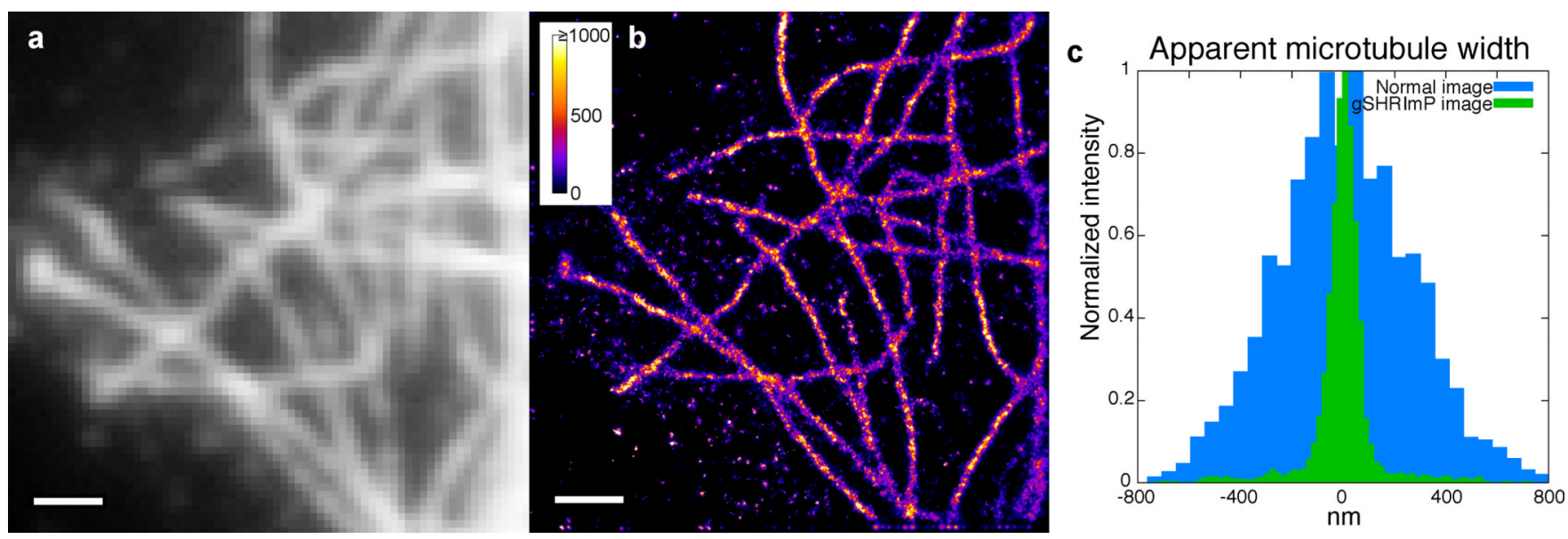

Figure 3.

Application of gSHRImP to photobleaching microtubules labeled with CF633 secondary antibodies in fixed COS-7 cells. (a) Normal fluorescence image. (b) gSHRImP image (maximum-likelihood 2-d Gaussian fitting; average localization error $=17 \mathrm{~nm}$ ). Pixel length $=10 \mathrm{~nm}$. (c) Comparison of the microtubule FWHMs (least-squares elliptical Gaussian fitting; average spot localization error $=21 \mathrm{~nm}$ ). In the normal image, microtubule width at FWHM of the intensity $=500 \mathrm{~nm}$; gSHRImP image, FWHM $=100 \mathrm{~nm}$. Scale bar $=1 \mu \mathrm{m}$. 

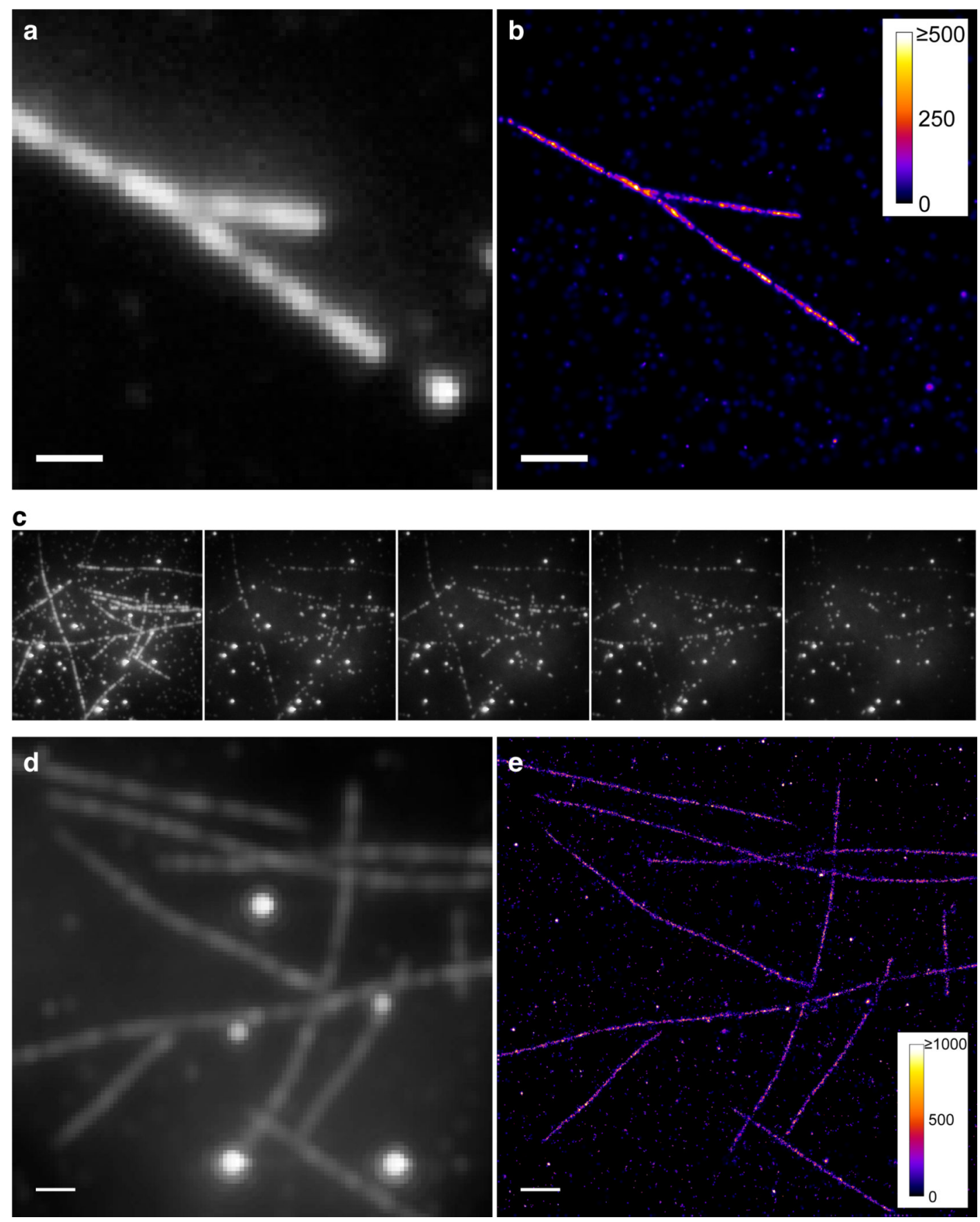

Figure 4.

Diffusive and transient labeling of microtubules on glass. (a-b) Labeling using Oregon Green 488 paclitaxel in solution. (a) Normal fluorescence image (frame \#1 of image sequence). (b) gSHRImP image. Individual spots were localized with an average $33 \mathrm{~nm}$ localization error. Pixel length $=10 \mathrm{~nm}$. (c-e) Transient labeling using streptavidin S45A. (c) Image sequence with frames selected at intervals of 499 frames. (d) Average of all frames in the image stack. Very bright spots are fluorescent $40 \mathrm{~nm}$ dark-red beads (Invitrogen, Cat. no. F-8789), which were used as fiduciary markers. (e) gSHRImP image. Average spot localization error $=9 \mathrm{~nm}$. Frame acquisition time was $0.5 \mathrm{~s}$. 2000 frames were analyzed. Pixel length $=10 \mathrm{~nm}$. Scale bar $=1 \mu \mathrm{m}$. 

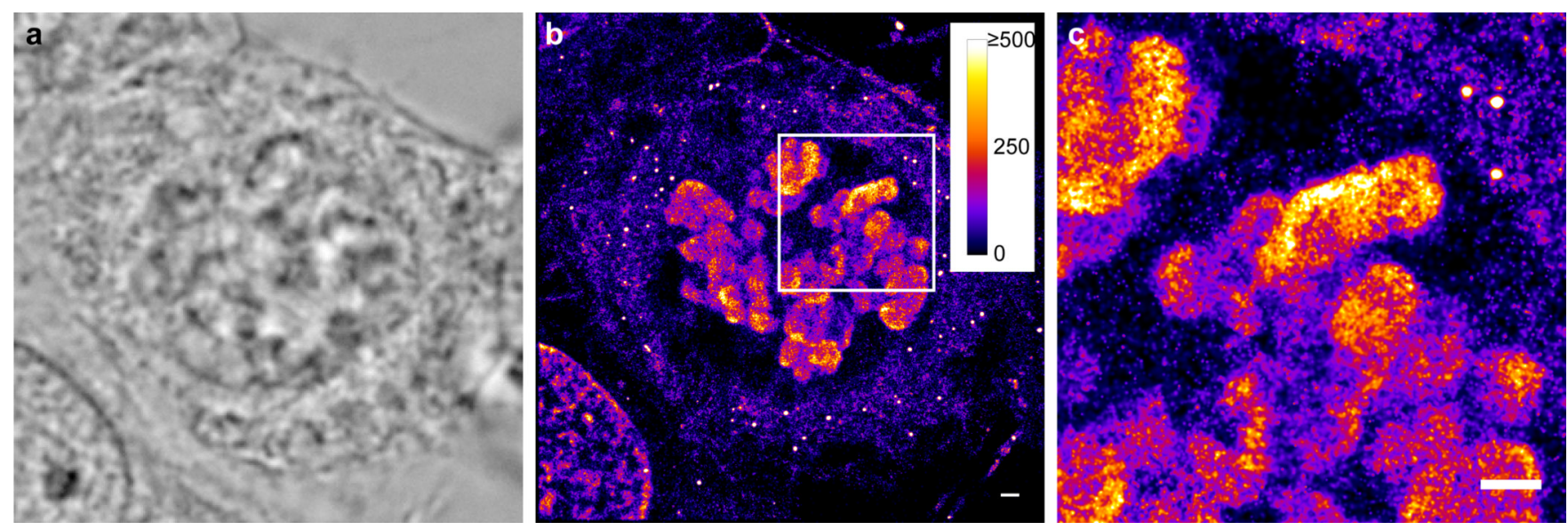

Figure 5.

Chromosomal DNA imaging in HeLa cells. The cells were imaged in $50 \mathrm{nM}$ POPO-3.

RNase was added to reduce POPO-3 binding to RNA. The objective's focus was adjusted once during acquisition. Frames were taken at $10 \mathrm{~Hz}$ for 30000 frames. The center cell shown is identified as being in prophase. (a) Bright field image. (b) gSHRImP image. Average plotted fluorophore localization error $=33 \mathrm{~nm}$. Pixel size $=10 \mathrm{~nm}$. Very bright spots near periphery are due to quantum dots, which were added for estimating stage drift. (c) Zoomed in portion of (b). Scale bar $=1 \mu \mathrm{m}$. 